\title{
The distribution of COPD in UK general practice using the new GOLD classification
}

\author{
John Haughney ${ }^{1}$, Kevin Gruffydd-Jones², June Roberts ${ }^{3}$, Amanda J. Lee $^{4}$, \\ Alison Hardwell ${ }^{5}$ and Lorcan McGarvey ${ }^{6}$
}

Affiliations: ${ }^{1}$ Centre of Academic Primary Care, University of Aberdeen, Aberdeen, ${ }^{2}$ Box Surgery, Corsham, ${ }^{3}$ Salford Royal NHS Foundation Trust, Salford, "Medical Statistics Team, University of Aberdeen, Aberdeen, ${ }^{5}$ National Services for Health Improvement, Dartford, and ${ }^{6}$ Centre of Infection and Immunity, The Queen's University of Belfast, Belfast, UK.

Correspondence: J. Haughney, Centre of Academic Primary Care, University of Aberdeen, Polwarth Building, Foresterhill Road, Aberdeen, AB25 2ZD, UK. E-mail: j.haughneyवabdn.ac.uk

ABSTRACT The new Global Initiative for Chronic Obstructive Lung Disease (GOLD) 2011 document recommends a combined assessment of chronic obstructive pulmonary disease (COPD) based on current symptoms and future risk.

A large database of primary-care COPD patients across the UK was used to determine COPD distribution and characteristics according to the new GOLD classification. 80 general practices provided patients with a Read code diagnosis of COPD. Electronic and hand searches of patient medical records were undertaken, optimising data capture.

Data for 9219 COPD patients were collected. For the 6283 patients with both forced expiratory volume in $1 \mathrm{~s}$ (FEV1) and modified Medical Research Council scores (mean $\pm \mathrm{SD}$ age $69.2 \pm 10.6$ years, body mass index $27.3 \pm 6.2 \mathrm{~kg} \cdot \mathrm{m}^{-2}$ ), GOLD 2011 group distributions were: A (low risk and fewer symptoms) $36.1 \%$, B (low risk and more symptoms) $19.1 \%, \mathrm{C}$ (high risk and fewer symptoms) $19.6 \%$ and D (high risk and more symptoms) $25.3 \%$. This is in contrast with GOLD 2007 stage classification: I (mild) $17.1 \%$, II (moderate) $52.2 \%$, III (severe) $25.5 \%$ and IV (very severe) $5.2 \%$. 20\% of patients with FEV1 $\geqslant 50 \%$ predicted had more than two exacerbations in the previous 12 months. $70 \%$ of patients with $\mathrm{FEV} 1<50 \%$ pred had fewer than two exacerbations in the previous 12 months.

This database, representative of UK primary-care COPD patients, identified greater proportions of patients in the mildest and most severe categories upon comparing 2011 versus 2007 GOLD classifications. Discordance between airflow limitation severity and exacerbation risk was observed.

@ERSpublications

GOLD 2011 COPD classification criteria identified more patients in the mildest and more severe groups than GOLD 2007 http://ow.ly/t4uiO

For editorial comments see page 949.

This article has supplementary material available from www.erj.ersjournals.com

Received: April 122013 | Accepted after revision: Sept 122013 | First published online: Oct 312013

Clinical trial: This study is registered at www.clinicaltrials.gov with identifier number NCT01263340.

Support statement: This study was undertaken by the National Service for Health Improvement using an unrestricted educational grant, funded by GlaxoSmithKline.

Conflict of interest: Disclosures can be found alongside the online version of this article at www.erj.ersjournals.com

Copyright @ERS 2014 


\section{Introduction}

Chronic obstructive pulmonary disease (COPD) is a multicomponent disease that shows marked heterogeneity in terms of clinical outcomes, disease severity and progression. Traditionally, the severity of COPD has been assessed by the degree of airflow obstruction, using a staging system based on forced expiratory volume in $1 \mathrm{~s}(\mathrm{FEV} 1)$ [1]. It is now recognised that FEV1 alone fails to represent the complexity of COPD and is poorly related to important patient factors including breathlessness, health status, level of comorbidities and exacerbation risk [2,3]. This deficiency prompted the UK National Institute for Health and Care Excellence to recommend the multidimensional assessment of COPD severity as a key area for implementation [4]. Most recently, the Global Initiative for Chronic Obstructive Lung Disease (GOLD) 2011 strategy document proposed a combined assessment of COPD using the modified Medical Research Council (mMRC) dyspnoea grade, current health status assessed by the COPD Assessment Test (CAT) and future risk based on either severity of airflow limitation or exacerbation history [5]. The new assessment categorises patients into one of four groups, as follows. A: low risk and fewer symptoms; B: low risk and more symptoms; C: high risk and fewer symptoms; and D: high risk and more symptoms.

Only a few studies have reported on the utility of the new GOLD 2011 classification in COPD populations. In an analysis of COPD patients $(n=6628)$ drawn from two large general population surveys, LANGE et al. [6] found the new classification to have improved prognostic capacity to predict future exacerbation risk compared with the older version. They also reported that patients with more symptoms (dyspnoea) but relatively well preserved lung function (subgroup B) had poorer survival than those with more severe obstruction but fewer symptoms (subgroup C), highlighting the existence of a number of distinctly different disease phenotypes with regard to future patient risk. Using a dataset of 1041 COPD patients (approximately one-third from primary care and two-thirds from specialist care), NADEAU et al. [7] reported that a fifth of patients considered "low risk" using the new GOLD classification had suffered an exacerbation in the previous year. As a history of a COPD exacerbation in the previous year is now recognised as a strong predictor of future exacerbation risk [8], this highlights potential limitations of the new classification system. Additional considerations that need addressing include identifying the optimal cut-points on the mMRC and CAT tools for symptomatic subgrouping of patients. Using data from European primary-care COPD patients $(n=1810)$, JONES et al. [9] suggested that a mMRC cut-point of $\geqslant 1$ rather than $\geqslant 2$ had the closest equivalence to a CAT cut-point of $\geqslant 10$.

Therefore, although the new GOLD classification may need refinement in future, it is clear that multidimensional assessment of COPD patients has important clinical implications. This is particularly the case in primary care where the majority of COPD patients are managed clinically. To determine the true distribution of COPD in primary care according to the newly recommended GOLD classification requires analysis of large and representative primary-care databases. However, they may be limited by incomplete data on important variables including lung function, accurate recording of exacerbations, comorbidities and details of contacts with external agencies (hospital admissions, outpatient visits or out-of-hours consultations).

In an attempt to overcome some of these limitations we have assembled a large and representative database of COPD patients attending primary care throughout the UK. Here, we utilise these data to report the distribution of COPD severity across the UK using the new GOLD 2011 assessment framework and its comparison with the previous GOLD 2007 classification.

\section{Methods}

\section{Study design and study population}

This was an observational, multicentre, retrospective cohort study which aimed to evaluate prevalence, incidence, severity, comorbidities and burden of disease in patients with COPD. Patients were identified by the presence of a Read code diagnosis (the standard UK diagnosis classification system) for COPD [10]. Patients having a diagnosis of COPD for $\geqslant 1$ year were eligible for inclusion; where available, data for the past 3 years were collected.

The database used was generated by the National Service for Health Improvement (NSHI) and sponsored by GlaxoSmithKline. General practices across the UK were invited to participate to ensure a broad representation of practices and COPD patients. NSHI nurse specialists performed electronic searches of electronic patient records using MIQUEST (Morbidity Information Query and Export Syntax) software for collecting data from general practice computer systems [11] on identified COPD patients. This was supplemented by a hand search of all patient records held by each practice (see online supplementary material for further details of sampling procedure and database generation).

Consent was obtained from each general practice. Ethics approval was not required for this study as there was no patient contact and no patient-identifiable material was recorded; all data collected on the database were anonymised. 


\section{Study outcomes}

Demographic characteristics

Data collection items included: sex, age, height, weight, body mass index (BMI), duration of COPD, smoking history, concurrent therapies for COPD and comorbidities. Comorbidities of common interest were cardiovascular disease, cerebrovascular disease, cancer, diabetes mellitus, depression and osteoporosis; defined as an ever-recorded diagnosis.

FEV1

The most recently recorded spirometry readings available to the practice were documented. The severity of airflow limitation categories was defined according to GOLD 2007 criteria: I (mild): FEV1 $\geqslant 80 \%$ predicted; II (moderate): FEV1 50-79\% pred; III (severe): FEV1 30-49\% pred; and IV (very severe): FEV1 <30\% pred [1].

\section{COPD exacerbations}

Exacerbations were identified by researcher scrutiny of physicians' records (primary, secondary or emergency care) and this evidence was cross-checked by the identification of one or more of the following: 1) Read code in the clinical records; 2) prescription of oral corticosteroids in primary care for an intended duration of $\geqslant 3$ days, where no other reason for the prescription other than the presence of COPD was identified; 3) attendance at an out-of-hours or emergency centre or accident and emergency department with a primary diagnosis of COPD resulting in a prescription of oral corticosteroids for an intended duration of $\geqslant 3$ days; or 4 ) a hospital admission with a primary diagnosis of COPD.

\section{Symptom assessment}

Where available, the most recently recorded score for mMRC was documented. The mMRC dyspnoea scale describes five grades of breathlessness ranging from 0 (least severe breathlessness) to 4 (most severe breathlessness) [12]. It was noted if a CAT score was available, although its magnitude was not recorded. The CAT is an eight-item health status questionnaire with total score ranging from 0 (best) to 40 (worst) [13].

\section{Statistical analysis}

Data were entered into SPSS (version 21; IBM, Armonk, NY, USA) for analyses. Summary statistics included the mean $\pm S D$ or median and interquartile range (IQR) for continuous data and number (percentage) for categorical data. Demographic and clinical factors were compared across GOLD 2007/2011 groups using the Chi-squared test for categorical data and ANOVA for continuous data. A p-value of $\leqslant 0.05$ was used to denote statistical significance throughout.

\section{Results}

General practice sample characteristics

In total, 80 practices across the UK contributed to this study and represented most geographical areas. 32 $(40 \%)$ of the practices were training practices; the majority $(90 \%)$ of practices had six general practitioners (GPs) or fewer and $24(30 \%)$ practices had two or fewer GPs. More details of the sample characteristics can be found in the online supplementary material.

\section{Study population}

The total patient population of all practices was 540793 ; data on 9219 patients with a Read code diagnosis of COPD were collected between November 2010 and January 2012 (fig. 1), giving an overall prevalence of COPD of $1.7 \%$. Across the 80 practices, COPD prevalence varied from $0.3 \%$ to $4.3 \%$ with a median (IQR) practice prevalence of $1.8 \%(1.2-2.3 \%)$.

The mean \pm SD age of the group was $69.5 \pm 11.1$ years, approximately half were male, most were either former $(53 \%)$ or current $(37 \%)$ smokers and mean \pm SD BMI for the group was $27.2 \pm 6.3 \mathrm{~kg} \cdot \mathrm{m}^{-2}$ (table 1). According to the GOLD spirometry-based severity criteria, patients were most commonly classified as having moderate COPD (52\%), with very few patients being classified as having very severe COPD (5\%). The mean annual rate of exacerbations in the previous 12 months was 0.89 ; just under a half of the patients had experienced at least one exacerbation in the previous year and approximately one-fifth had undergone two or more exacerbations.

A score for mMRC was recorded for 7119 (77\%) patients. The most common scores were 1 (breathless when hurrying on level or up a slight hill) $(37.0 \%)$ and 2 (walk slower than people of same age on the level due to breathlessness or stop for breath when walking on the level at own pace) (27.4\%). A total of 278 (3\%) 
patients in 10 practices had a CAT score recorded. Due to the small number available, no further analysis was undertaken.

\section{Comorbidities}

Approximately 79\% $(\mathrm{n}=7267)$ of patients had one or more comorbidities. The most commonly reported comorbidities, defined as an ever-recorded diagnosis, were cardiovascular disease $(46.3 \%)$ followed by asthma $(23.3 \%)$ and cerebrovascular disease (16.6\%). Depression, cancer and diabetes were each reported by $\sim 14-15 \%$ of the population.

\section{Medication}

The proportion of patients receiving any COPD medication was $87.4 \%$. The most commonly prescribed maintenance therapies were combined treatment with an inhaled corticosteroid plus long-acting $\beta$-agonist $(55.8 \%)$ and the long-acting anticholinergic tiotropium (46.3\%).

\section{Demographic and clinical characteristics by the 2007 and 2011 GOLD classifications}

Patient characteristics according to the GOLD 2011 and 2007 classifications are shown in table 2.

By GOLD 2007 classification, patients in the subgroups with greatest airflow imitation (III and IV) were older, more likely to be male and have lower BMI compared with patients with GOLD stage I and II. Patients in GOLD stage II and III had higher levels of cardiovascular comorbidities compared with stages I and IV. The proportions of patients with lung cancer increased with increasing severity of airflow limitation.

When classified by the GOLD 2011 criteria, patients in the higher symptom subgroups (B and D) were older and had higher levels of comorbidities compared with the low symptom subgroups (A and C). Patients in group B (low risk with preserved lung function but more symptoms) were more likely to be female, have the highest BMI and the greatest proportion with cardiovascular comorbidities. The proportions of patients with one or more COPD exacerbation by subgroups A-D were: A: $65.0 \%$, B: $71.5 \%, \mathrm{C}: 85.6 \%$, and D: $88.5 \%$; and those with one or more hospitalisation were: A: $4.0 \%$, B: $8.3 \%$, C: $13.2 \%$, and D: $26.4 \%$. The proportions of patients who were prescribed an inhaled steroid preparation (either a monotherapy or as combination therapy) were: A: $55.8 \%$, B: $70.9 \%$, C: $76.5 \%$, and D: $89.6 \%$.

\section{Distribution of patients according to new GOLD categories}

The distribution of patients into the new GOLD 2011 categories, using patients with both a valid FEV1\% pred and a valid mMRC score $(n=6283)$ is presented in figure $2 a$. The largest proportion of patients $(36 \%)$ was classified into subgroup A (low risk and fewer symptoms), approximately a fifth of patients were classified into subgroups B (low risk and more symptoms, 19.1\%) and C (high risk and fewer symptoms, 19.6\%), and approximately a quarter (25.3\%) were classified into subgroup D (high risk and more symptoms).

Therefore, this new GOLD classification categorised $46 \%$ of the population as "high risk" whereas only $30.5 \%$ of the population would be considered high risk (FEV $1<50 \%$ GOLD stage III and IV) according to the GOLD 2007 system. The distribution of patients in primary care using the new GOLD 2011 classification suggests a shift towards two distinct subgroups: A (low risk and fewer symptoms) and D (high risk and more symptoms). Such a pattern of distribution is not as evident using the GOLD 2007 classification (fig. 3).

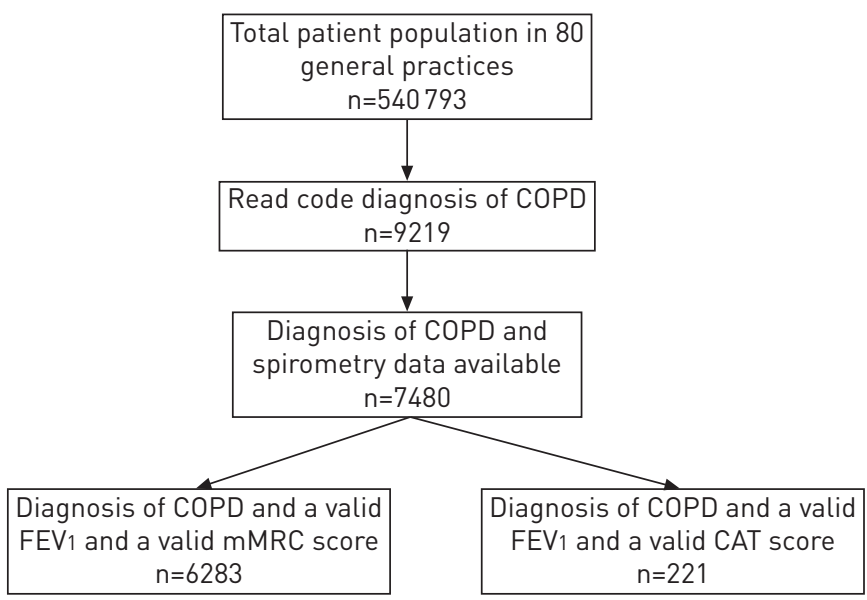

FIGURE 1 Study flow according to patient data. COPD: chronic obstructive pulmonary disease; FEV1: forced expiratory volume in $1 \mathrm{~s}$; mMRC: modified Medical Research Council; CAT: COPD Assessment Test. 
TABLE 1 Demographic and baseline characteristics of chronic obstructive pulmonary disease (COPD) cohort

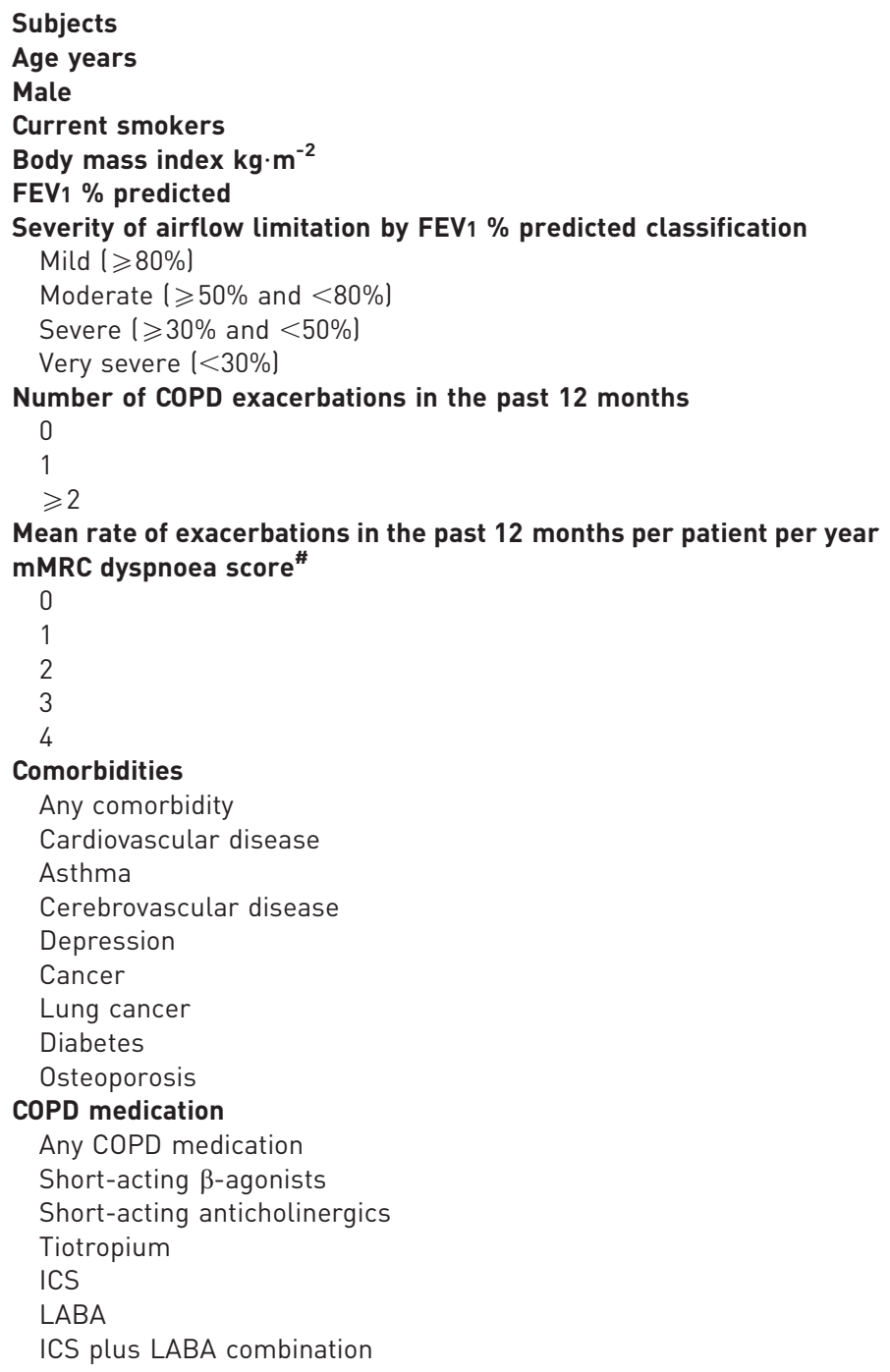

Data are presented as $n$, mean \pm SD or $n(\%)$. Percentages are calculated from the number of valid answers to each individual characteristic and, due to missing values, may not necessarily be based on the entire cohort of 9219. FEV1: forced expiratory volume in $1 \mathrm{~s}$; mMRC: modified Medical Research Council; ICS: inhaled corticosteroids; LABA: long-acting $\beta$-agonists. " : MRC grades 1-5 were captured, these are equivalent land have identical wordingl to mMRC $0-4$, as follows. 0 : only breathless with strenuous exercise; 1 : breathless when hurrying on the level or up a slight hill; 2: walk slower than people of same age on the level due to breathlessness or stop for breath when walking on level at own pace; 3: stop for breath after walking 100 yards or a few minutes on the level; and 4: too breathless to leave house or breathless when dressing.

The GOLD 2013 guidelines recommend that one or more hospitalisations for COPD exacerbations should be considered high risk. The results of applying GOLD 2013 to our population is shown in figure 2 b.

Comparison of risk stratification by exacerbation frequency and by airflow limitation

For the 7480 patients with a valid FEV1 \% pred, the proportion of patients categorised as high risk differed according to risk assessment used (fig. 4). Of the 5198 patients who had low-risk airflow limitation, 1029 (20\%) had had two or more exacerbations in the previous 12 months. Of the patients with high-risk airflow limitation $(n=2282), 1607(70 \%)$ had a low exacerbation risk profile (one or fewer exacerbations). Similarly, for patients with a high exacerbation risk $(n=1704), 1029(60 \%)$ had FEV1 $\geqslant 50 \%$ pred. 


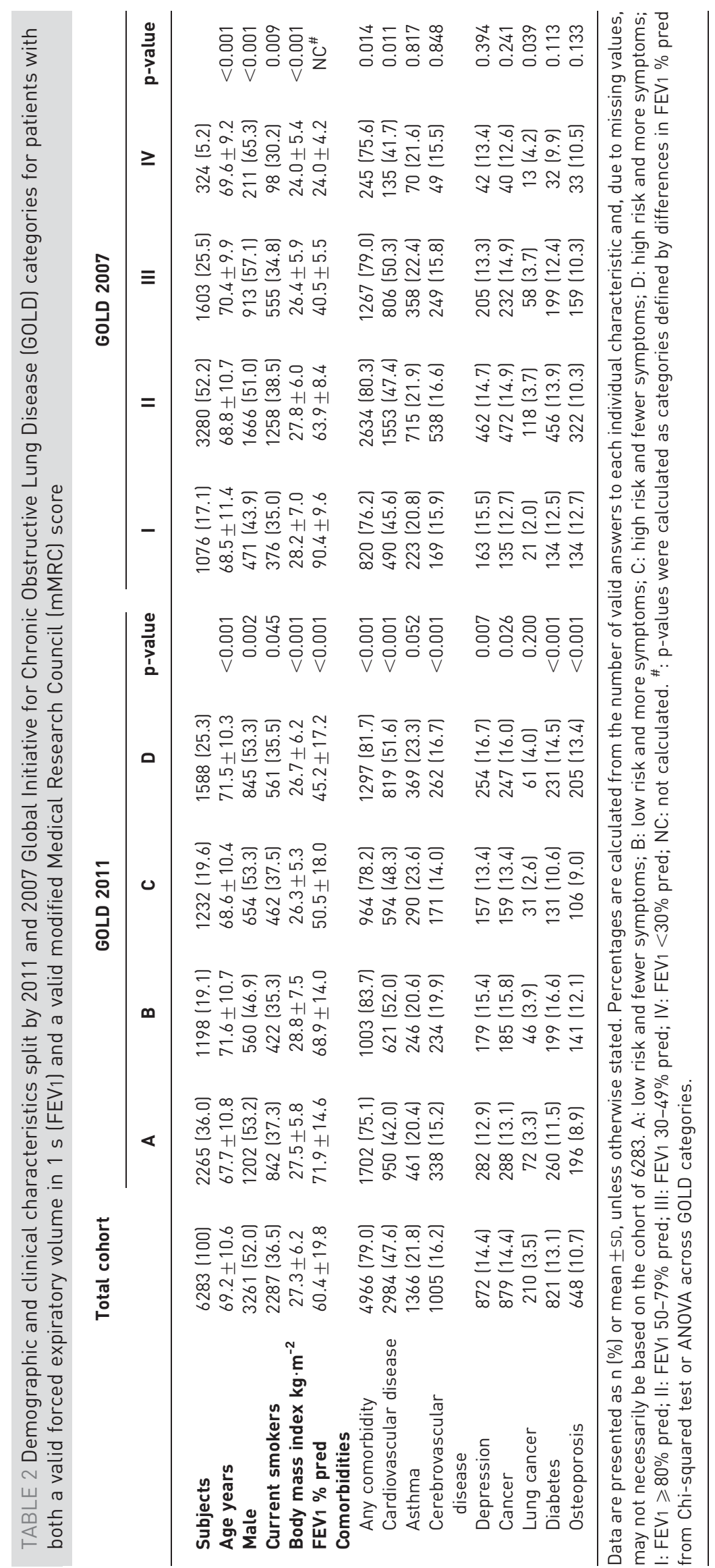



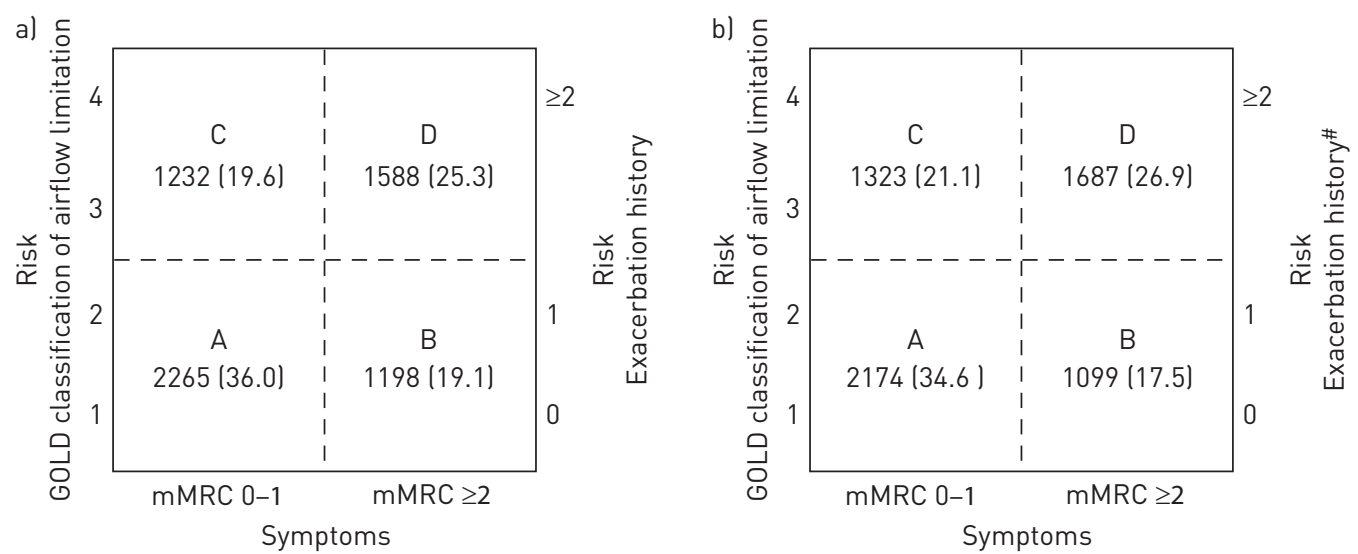

FIGURE 2 Distribution of chronic obstructive pulmonary disease (COPD) patients using a) the 2011 Global Initiative for Chronic Obstructive Pulmonary Disease (GOLD) categories and b) the 2013 GOLD categories. Data are presented as $\mathrm{n}(\%)$ and includes patients with both a valid forced expiratory volume in $1 \mathrm{~s}$ and a valid modified Medical Research Council (mMRC) score. $\mathrm{n}=6283$. Categories, A: low risk and fewer symptoms; B: low risk and more symptoms; C: high risk and fewer symptoms; D: high risk and more symptoms. ${ }^{\#}: \geqslant 1$ hospitalisations due to COPD exacerbations was considered high risk.

\section{Discussion}

Our study is the first to describe the distribution of COPD patients in primary care throughout the UK, according to the GOLD 2011 classification. Our key findings are that a greater proportion of COPD patients were identified as being at high risk of adverse health outcomes than would otherwise have been determined by the previous GOLD classification; the subgroups with higher symptom levels (B and D) were associated with higher levels of comorbidities compared with the low symptom level subgroups A and C. There was a large heterogeneity in patient risk, whether determined by FEV1 \% pred or exacerbation history.

In our study we have shown that, compared with the GOLD 2007 classification, the GOLD 2011 categories had more patients in both the mildest (group A) and most severe groups (group D). Similarly, LANGE et al. [6] reported a higher proportion of patients in the most severe group when comparing the GOLD 2011 (4.5\%) and GOLD $2007(0.7 \%)$ classifications. In contrast, a higher proportion of subjects (77\%) were categorised into group A compared with our own findings (36\%), reflecting a major difference in the study populations. The study by LANGE et al. [6] was a general population study comprising a large proportion of treatment-naïve patients in contrast to our own population of already diagnosed, largely treated COPD patients. The distribution of our patients into groups A-D was comparable to the findings of NADEAU et al. [7], although their population was skewed towards specialist care rather than primary care. Using the GOLD 2011 classification on the Health-Related Quality of Life in COPD in Europe Study (HEED) database JONES et al. [9] reported greater proportions of patients in the higher risk subgroups C and D compared to ours, probably reflecting distinct clinical differences in study population; the HEED database captured patients presenting to their primary-care physician for a scheduled visit, with $13 \%$ presenting with a COPD exacerbation [14].

Our dataset provides further evidence of the difficulty in classifying disease risk in COPD. One-fifth of patients had experienced two or more exacerbations in the previous year despite having "low-risk" airflow limitation (FEV1 $>50 \%$ pred), and the majority of patients $(70 \%)$ with "high-risk" airflow limitation (FEV1 $<50 \%$ pred) had no or one exacerbation in the previous year. Although some studies suggest that there is an increased risk of exacerbations with increased levels of airflow limitation [5, 15], these data endorse the evidence that lung function alone does not predict the likelihood of having an exacerbation. Our findings agree with the Evaluation of COPD Longitudinally to Identify Predictive Surrogate Endpoints (ECLIPSE) study [3], which showed a heterogeneous response in the rate of exacerbations across all GOLD stages. LANGE et al. [6] also reported heterogeneity in GOLD groups C and D with respect to risk of future exacerbations, and, perhaps surprisingly, showed that patients in group B, characterised by more severe dyspnoea, had worse survival outcomes than patients in group $\mathrm{C}$ with greater airflow limitation.

LANGE et al. [6] speculated that the poorer survival outcomes may have been related to the higher incidence of cardiovascular comorbidities in group B compared with group C. The characteristics of patients in the GOLD 2011 subgroups showed marked similarities in our study and the LANGE et al. [6] study. In both, the patients in subgroups B and D were older and had higher levels of cardiovascular comorbidities. In our study, 


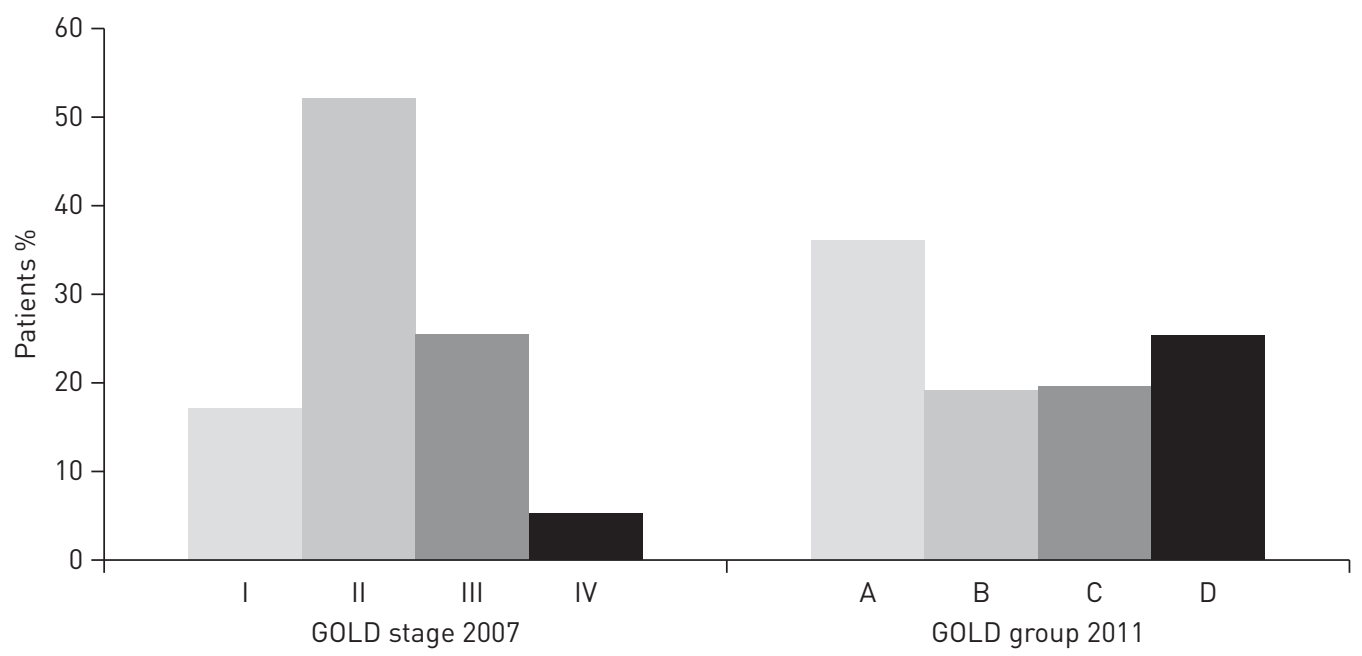

FIGURE 3 Comparison of the distribution of patients using the Global Initiative for Chronic Obstructive Pulmonary Disease (GOLD) stages I-IV 2007 and GOLD groups A-D 2011 classifications. Includes patients with both valid forced expiratory volume in $1 \mathrm{~s}$ and valid modified Medical Research Council scores. $\mathrm{n}=6283$.

patients in subgroups B and D had higher levels of cerebrovascular disease, depression, cancer and diabetes compared with patients in subgroups A and C. We showed no clear pattern between GOLD 2007 categories and level of comorbidities, which was consistent with findings in the ECLIPSE study [3]. Both our study and the LANGE et al. [6] study suggest that subgroup B patients warrant close attention; their symptoms possibly being driven by their associated comorbidities, in particular cardiovascular disease.

We report a prevalence of COPD of $1.7 \%$ which compares well with the reported mean prevalence of $1.7 \%$ for the UK in 2012, from a UK Quality and Outcomes Framework NHS database [16]. Our study did not evaluate specific age groups and may explain the lower reported prevalence. The differences may also be related to methods of reporting, i.e. prevalence based on diagnosed and treated COPD compared with that based on spirometry measurements alone. COPD prevalence figures do, of course, vary considerably depending on the age range of the population included. For example, UK figures report a prevalence of $4.1 \%$ in adults aged $\geqslant 30$ years [17] and $13.3 \%$ in adults aged $>35$ years [18]. A review of prevalence data across Europe reported prevalences from $2.1 \%$ to $26.1 \%$, depending on country, age group and methods of assessment used [19].

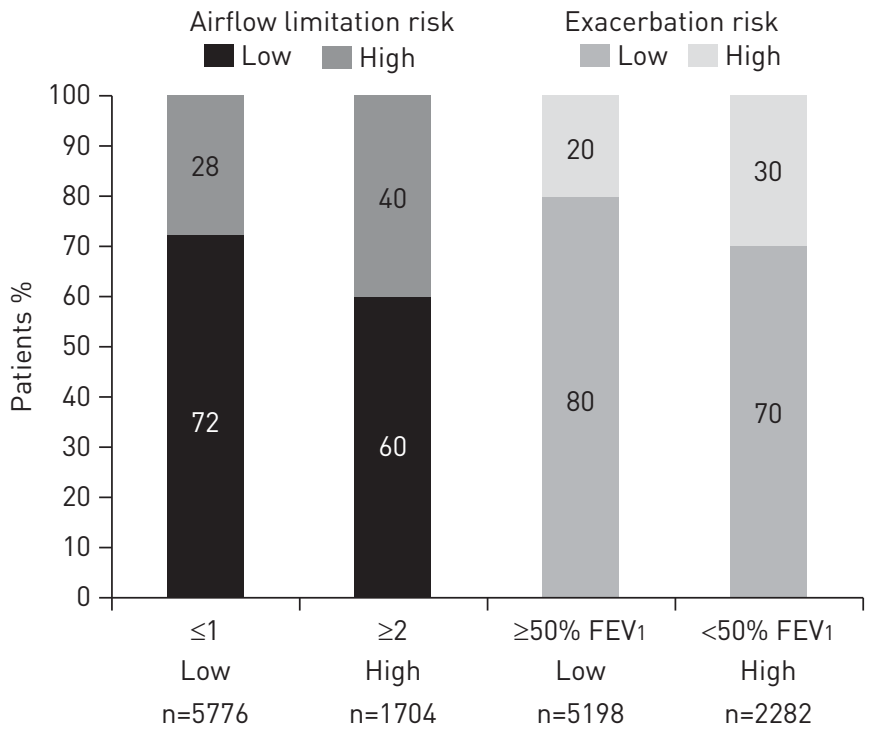

FIGURE 4 Overlap of risk categories (airflow limitation and exacerbation history). The data include patients with a valid forced expiratory volume in $1 \mathrm{~s}$ (FEV1). $\mathrm{n}=7480$. 
For this study we sought to ensure the dataset generated was representative of the primary care throughout the UK. Specifically, we recruited general practices across a broad geographical distribution and ensured that both the variation in practice size and the proportion with a primary-care training designation was reflective of general practices in the UK. A particular strength of this database is its size, and, due to the comprehensive searches conducted, there were very few missing data items for demographic characteristics and relatively high numbers of records for clinical outcomes, thus providing a reliable source of data for exacerbations, FEV1, mMRC scores and comorbidities. Although the quality of FEV1 recordings could not be determined, the data represent real-world data capture. We acknowledge that findings from this study cannot be extrapolated to other European countries due to differences in healthcare systems, including the provision of primary care. Another limitation is the limited CAT data that was collected in only $3 \%$ of patients across a few practices. However, these data are representative of primary-care practice and reflect the fact that this health status measure is not yet routinely collected in primary care. In addition, the new GOLD classification allows the assessment of symptoms based on mMRC or CAT scores, and the optimal symptom questionnaire and associated cut-points are still the subject of some debate [9].

The new GOLD strategy combined assessment brings a welcome and marked change in the approach to managing COPD patients, which considers both the disease impact (current symptoms and activity limitation) and the future risk of disease progression (especially exacerbations). This may have important implications for patients in primary care in terms of treatment and their level of primary-care contact. For clinicians, resources can be targeted to patients with the greatest needs in terms of pharmacological therapy, treatment of comorbidities and frequency of monitoring required. For patients this should result in improved and targeted care which should maximise the treatment of symptoms and minimise the risk of exacerbations.

In conclusion, this study successfully used a comprehensive database, representative of COPD patients across the UK, and showed that classifying patients using the new GOLD 2011 criteria identified larger proportions of patients in the mildest and more severe groups compared with the GOLD 2007 classification. We also identified discordance between the level of airflow limitation and exacerbation risk, highlighting the importance of recognising distinct COPD phenotypes. The GOLD 2011 classification should help primarycare physicians target patients for pharmacological therapy and identify those in need of more frequent monitoring to reduce future long-term risks.

\section{Acknowledgements}

The authors would like to thank the participating GP practices for their contribution to this study. The authors would also like to acknowledge editorial support in the form of assistance in the development of the manuscript drafts, including assembling tables and figures, collating author comments and copyediting, which was provided by K. Hollingworth of Continuous Improvement Ltd (Kingsbridge, UK). This support was funded by NSHI.

\section{References}

1 Rabe KF, Hurd S, Anzueto A, et al. Global strategy for the diagnosis, management, and prevention of chronic obstructive pulmonary disease: GOLD executive summary. Am J Respir Crit Care Med 2007; 176: 532-555.

2 Jones PW, Agusti AGN. Outcomes and markers in the assessment of chronic obstructive pulmonary disease. Eur Respir J 2006; 27: 822-832.

3 Agusti A, Calverley PMA, Celli B, et al. Characterisation of COPD heterogeneity in the ECLIPSE cohort. Respir Res 2010; 11: 122 .

4 NICE National Institute for Health and Care Excellence. Chronic obstructive pulmonary disease: management of chronic obstructive pulmonary disease in adults in primary and secondary care. 2010. http://publications.nice.org. uk/chronic-obstructive-pulmonary-disease-cg101 Date last accessed: March 7, 2013. Date last updated: 2010.

5 Vestbo J, Hurd SS, Agustí AG, et al. Global strategy for the diagnosis, management and prevention of chronic obstructive pulmonary disease: GOLD executive summary. Am J Respir Crit Care Med 2013; 187: 347-365.

6 Lange P, Marott JL, Vestbo J, et al. Prediction of the clinical course of chronic obstructive pulmonary disease, using the new GOLD classification: a study of the general population. Am J Respir Crit Care Med 2012; 186: 975-981.

7 Nadeau G, Adamek L, Small M. Distribution of COPD patients in the GOLD assessment framework by exacerbations. Eur Respir J 2012; 40: Suppl, 56. p980.

8 Hurst JR, Vestbo J, Anzueto A, et al. Susceptibility to exacerbation in chronic obstructive pulmonary disease. N Engl J Med 2010; 363: 1128-1138.

9 Jones PW, Adamek L, Nadeau G, et al. Comparisons of health status scores with MRC grades in COPD: implications for the GOLD 2011 classification. Eur Respir J 2013; 42: 647-654.

10 NHS Connecting for Health Read Codes. www.connectingforhealth.nhs.uk/systemsandservices/data/uktc/readcodes Date last accessed: March 7, 2013. Date last updated: April 1, 2013.

11 NHS Connecting for Health MIQUEST. www.connectingforhealth.nhs.uk/systemsandservices/data/miquest Date last accessed: March 7, 2013. Date last updated: November 1, 2012.

12 Bestall JC, Paul EA, Garrod R, et al. Usefulness of the Medical Research Council (MRC) dyspnoea scale as a measure of disability in patients with chronic obstructive pulmonary disease. Thorax 1999; 54: 581-586.

13 Jones PW, Harding G, Berry P, et al. Development and first validation of the COPD Assessment Test. Eur Respir J 2009; 34: 648-654.

14 Jones PW, Brusselle G, Dal Negro RW, et al. Health-related quality of life in patients by COPD severity within primary care in Europe. Respir Med 2011; 1: 57-66. 
15 Hoogendoorn M, Feenstra TL, Hoogenveen RT, et al. Association between lung function and exacerbation frequency in patients with COPD. Int J Chron Obstruct Pulmon Dis 2010; 5: 435-444.

16 Jamie G. Quality and Outcomes Framework NHS database. www.gpcontract.co.uk/browse/UK/12 Date last accessed: July 12, 2013. Date last updated: 2013.

17 Frank TL, Hazell ML, Linehan MF, et al. The estimated prevalence of chronic obstructive pulmonary disease in a general practice population. Prim Care Respir J 2007; 16: 169-173.

18 Shahab L, Jarvis MJ, Britton J, et al. Prevalence, diagnosis and relation to tobacco dependence of chronic obstructive pulmonary disease in a nationally representative population sample. Thorax 2006; 61: 1043-1047.

19 Atsou K, Chouaid C, Hejblum G. Variability of the chronic obstructive pulmonary disease key epidemiological data in Europe: systematic review. BMC Med 2011; 9: 7. 